\title{
5-Aminosalicylic acid intolerance is associated with a risk of adverse clinical outcomes and dysbiosis in patients with ulcerative colitis
}

\author{
Shinta Mizuno ${ }^{1 *}$, Keiko Ono $^{1 *}$, Yohei Mikami ${ }^{1}$, Makoto Naganuma ${ }^{1}$, Tomohiro Fukuda ${ }^{1}$, Kazuhiro Minami $^{1}$, \\ Tatsuhiro Masaoka ${ }^{1}$, Soichiro Terada ${ }^{2}$, Takeshi Yoshida ${ }^{3}$, Keiichiro Saigusa ${ }^{4}$, Norimichi Hirahara ${ }^{5}$, Hiroaki Miyata ${ }^{5}$, \\ Wataru Suda ${ }^{6}$, Masahira Hattori ${ }^{6}$, Takanori Kanai ${ }^{1}$ \\ ${ }^{1}$ Division of Gastroenterology and Hepatology, Department of Internal Medicine, Keio University School of Medicine, Tokyo; ${ }^{2}$ Endoscopy \\ Center, Edogawa Hospital, Tokyo; ${ }^{3}$ Department of Gastroenterology and Hepatology, Saitama Medical Center, Saitama, ${ }^{4}$ Department of \\ Gastroenterology and Hepatology, Tokyo Saiseikai Central Hospital, Tokyo; ${ }^{5}$ Department of Health Policy and Management, Keio University \\ School of Medicine, Tokyo; ${ }^{6}$ Laboratory for Microbiome Sciences, RIKEN Center for Integrative Medical Sciences, Kanagawa, Japan
}

Background/Aims: 5-Aminosalicylic acid (ASA) causes intolerance reactions in some patients. This study was performed to examine the prognosis of patients with ulcerative colitis (UC) and 5-ASA intolerance, and to evaluate the potential interaction between 5-ASA intolerance and the intestinal microbiota. Methods: We performed a retrospective cohort study of patients with UC who visited participating hospitals. The primary endpoint was to compare the incidence of hospitalization within 12 months between the 5-ASA intolerance group and the 5-ASA tolerance group. The secondary endpoint was to compare the risk of adverse clinical outcomes after the start of biologics between the 2 groups. We also assessed the correlation between 5-ASA intolerance and microbial change in an independently recruited cohort of patients with UC. Results: Of 793 patients, 59 (7.4\%) were assigned to the 5-ASA intolerance group and 734 (92.5\%) were assigned to the 5-ASA tolerance group. The admission rate and incidence of corticosteroid use were significantly higher in the intolerance than tolerance group $(P<0.001)$. In 108 patients undergoing treatment with anti-tumor necrosis factor biologics, 5-ASA intolerance increased the incidence of additional induction therapy after starting biologics $(P<0.001)$. The 5 -ASA intolerance group had a greater abundance of bacteria in the genera Faecalibacterium, Streptococcus, and Clostridium than the 5-ASA tolerance group $(P<0.05)$. Conclusions: In patients with UC, 5-ASA intolerance is associated with a risk of adverse clinical outcomes and dysbiosis. Bacterial therapeutic optimization of 5-ASA administration may be important for improving the prognosis of patients with UC. (Intest Res 2020;18:69-78)

Key Words: 5-Aminosalicylic acid; Colitis, ulcerative; Prognosis; Dysbiosis

\section{INTRODUCTION}

Ulcerative colitis (UC) is characterized by chronic intestinal inflammation, and is one of the 2 main subtypes of IBD. ${ }^{1}$ The

\footnotetext{
Received July 14, 2019. Revised November 4, 2019.

Accepted November 6, 2019.

Correspondence to Takanori Kanai, Division of Gastroenterology and

Hepatology, Keio University School of Medicine, 35 Shinanomachi, Shinjuku-

ku, Tokyo 160-8582, Japan. Tel: +81-3-3353-1211, Fax: +81-3-3341-3631,

E-mail: takagast@keio.jp

*These authors contributed equally to this study.
}

clinical outcomes of UC have been improved by recently developed therapeutic drugs, ${ }^{2,3}$ leading to reduced rates of surgical treatment and higher rates of clinical remission compared with previous conventional therapies. ${ }^{4,5}$ However, many of these newly developed therapeutic agents still have limited effectiveness, variable responses, potential adverse events caused by immunosuppression, and pharmacoeconomic issues. ${ }^{6}$

5-Aminosalicylic acid (ASA) consists of salazosulfapyridine and mesalazine. It is the first-line drug in induction therapy for mild to moderately active UC and in maintenance therapy for 
quiescent UC. ${ }^{7}$ Although 5-ASA causes relatively few serious adverse events, intolerance to 5-ASA occurs up to $15 \%$ of patients and their common clinical manifestations are diarrhea, fever, and rash..$^{8-11}$ However, the effect of 5-ASA intolerance on the prognosis of patients with UC has been reported in only a small number of case series; no large-scale studies have been conducted. Furthermore, the detailed mechanisms of 5-ASA intolerance have not been elucidated. Although it is presumed that allergic reactions, chemical toxicity, and chemical reactions are involved, allergic reactions have not been clearly distinguished from other types of reactions. Previous studies have revealed that the pathogenesis of UC involves many factors, such as immunological function, environmental factors, genetic predisposition, and the intestinal microbiota, ${ }^{12,13}$ and suggest that disturbance of the intestinal microbiota (dysbiosis) may be involved in 5-ASA intolerance. The present study was performed to clarify the optimal treatment strategy for patients with 5-ASA intolerance by investigating the relationship between 5-ASA intolerance and the prognosis in patients with UC. We also examined the relationship between 5-ASA intolerance and the intestinal microbiota in an attempt to devise a therapeutic strategy for resolving 5-ASA intolerance.

\section{METHODS}

\section{Medical Record Review Cohort (Keio IBD Longitudinal Cohort)}

We collected clinical information from our database of prospectively registered patients $(\mathrm{n}=827)$ with UC from January 1, 2015 to June 30, 2018 (Fig. 1). Using this database, we retrospectively reviewed the medical records of patients with UC who visited outpatient clinics at participating hospitals. 5-ASA intolerance was observed prior to hospitalization; therefore, hospitalizations were due to disease worsening in all cases. All participating hospitals are as follows; Keio University Hospital, Edogawa Hospital, Saitama Medical Center, Tokyo Saiseikai Central Hospital, Eiju General Hospital, and Shonan Keiiku Hospital.

\section{Microbial Analysis Cohort (Keio UC Microbial Cohort)}

In the microbial analysis, we recruited 181 patients with UC who were aged $\geq 20$ years and were in the remission phase (defined as a partial Mayo Clinic score of $\leq 1$ without bloody stool). As defined in previous reports, ${ }^{8,9,14}$ patients with 5-ASA intolerance were defined as patients who had experienced at

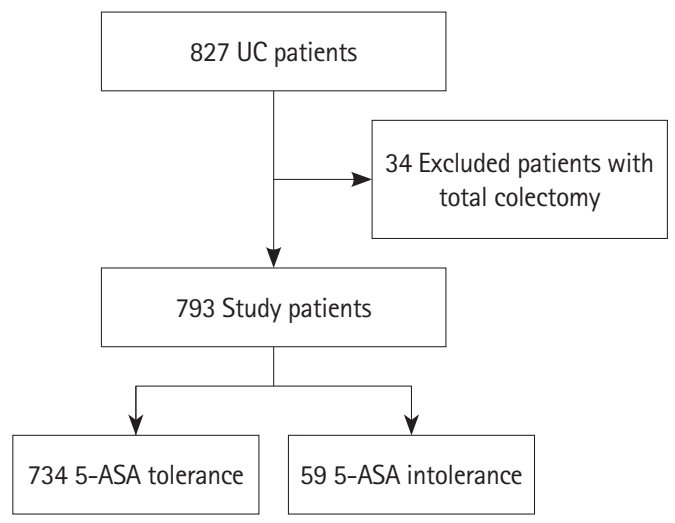

Fig. 1. Flowchart of participants in the risk analysis for admission divided into the 5-aminosalicylic acid (5-ASA) tolerance group and the 5-ASA intolerance group.

least one of the following symptoms in response to 5-ASA administration: headache, digestive symptoms, skin symptoms, or fever.

\section{End Points}

The primary endpoint in the Keio IBD Longitudinal Cohort was the incidence of hospitalization within 12 months, and the secondary endpoints were the incidence of hospitalization and the requirement for induction therapy (corticosteroids, calcineurin inhibitors, anti-TNF- $\alpha$ antibodies, and cytapheresis) between patients with 5-ASA intolerance (intolerance group) and those without 5-ASA intolerance (tolerance group).

The primary outcome in the Keio UC Microbial Cohort was alterations in the fecal microbiota composition.

\section{Ethical Considerations}

The medical record review (Keio IBD Longitudinal Cohort) was approved by the Ethics Committee of Keio University School of Medicine (approval No. 20160038). The study was registered at the University Hospital Medical Information Network Center (UMIN000027028), and was conducted in accordance with the principles of the Declaration of Helsinki. Because informed consent was not required for this retrospective observational study, we obtained the patients' consent for study inclusion by posting the study information in participating hospitals. All patient records and information were anonymized before the analysis.

The intestinal microbiota analysis (Keio UC Microbial Cohort) was approved by the Ethics Committee of Keio University School of Medicine (approval No. 20140101), and all participants provided written informed consent. 


\section{Statistical Analysis}

In the Keio IBD Longitudinal Cohort, the primary end point was compared between the intolerance and tolerance groups using the chi-square test. The secondary end points were compared using univariate analysis and logistic regression with multivariate analysis. The following variables were used as confounders in the logistic regression with multivariate analysis: age, disease duration, disease extent, serum albumin level, 5-ASA non-administration, 5-ASA intolerance, and immunomodulator (IM) tolerance. In the Keio UC Microbial Cohort, the primary outcome was compared between the intolerance and tolerance groups using Student $t$-test.

All statistical analyses were performed using SPSS software, version 25 (IBM Corp., Armonk, NY, USA). Two-sided $P$-values were considered significant at $<0.05$.

\section{Fecal Sample Collection}

Fecal samples were collected from patients just once. Fresh fecal samples were stored under anaerobic conditions in an AnaeroPack ${ }^{\mathrm{TM}}$ (Mitsubishi Gas Chemical Co., Inc., Tokyo, Japan) at $4^{\circ} \mathrm{C}$. Within 24 hours after sampling, liquid nitrogen was used to freeze the feces in $20 \%$ glycerol (Wako Pure Chemical Industries Ltd., Osaka, Japan) or phosphate-buffered saline solution (Life Technologies, Tokyo, Japan), and the samples were then stored at $-80^{\circ} \mathrm{C}$ until use.

\section{Bacterial DNA Extraction}

Bacterial DNA was prepared as previously reported. ${ }^{15}$ In brief, bacterial DNA was extracted by enzymatic lysis using lysozyme (Sigma-Aldrich Co. LCC, Tokyo, Japan) and achromopeptidase (Wako Pure Chemical Industries Ltd.). DNA samples were then purified by treatment with ribonuclease A (Wako Pure Chemical Industries Ltd.), followed by precipitation with 20\% polyethylene glycol solution (PEG6000 in $2.5 \mathrm{M}$ sodium chloride). DNA was then pelleted by centrifugation, rinsed with $75 \%$ ethanol, and dissolved in tris-ethylene diamine tetra acetic acid buffer.

\section{Sequencing and Processing of Bacterial 16S rRNA Genes from Fecal DNA}

Multiplexed 16S amplicon sequencing was performed on the MiSeq Illumina platform; the 16S rRNA gene V1-V2 region was amplified by PCR using the primers 27Fmod and $338 \mathrm{R}$ containing Illumina adaptor sequences and a unique 8-bp barcode. PCR amplicons were purified by AMPure XP magnetic purification beads (Beckman Coulter, Inc.), and quanti- fied using the Quant-iT PicoGreen dsDNA Assay Kit. An equal amount of each PCR amplicon was mixed and then sequenced using the MiSeq Reagent Kit v3 (600-cycles). Based on sample-specific barcodes, obtained reads were assigned to each sample, and paired-end reads were merged using the fastq-join program. We removed reads with an average quality value $<25$, mismatches to both universal primers, and possible chimeric reads. Among the high-quality reads, 3,000 reads per sample were randomly selected and grouped into operational taxonomic units (OTUs) using the UCLUST algorithm with a $96 \%$ identity threshold. Taxonomic assignments for each OTU were made by performing a similarity search against the public $16 \mathrm{~S}$ and NCBI (National Center for Biotechnology Information) genome databases using the GLSEARCH program. Assignments at the phylum, family, genus, and species levels were made using sequence similarity thresholds of $70 \%, 90 \%, 94 \%$, and $96 \%$, respectively. Downstream analyses and visualization were performed with $\mathrm{R}$ version 3.4.3 (R Foundation for Statistical Computing, Vienna, Austria) and heatmaps with hierarchically clustered rows, while columns were created using the pheatmap R package (https://cran.rproject.org/web/packages/pheatmap/index.html). Species with a minimal mean OTU > 30 were analyzed in the heatmap. All high-quality 16S V1-V2 sequences analyzed in the present study were deposited into the DNA Data Bank of Japan (DDBJ), GenBank (USA), and European Molecular Biology Laboratory (EMBL) databases.

\section{RESULTS}

\section{5-ASA Intolerance Increases the Risk of Hospitalization} During the study period, 827 patients with UC were registered in the IBD registry of our hospital. We excluded 34 patients who had undergone total colectomy and analyzed the remaining 793 patients. Table 1 shows the patients' characteristics, including therapeutic regimens. Of the 793 patients, 59 (7.4\%) were intolerant to 5-ASA (intolerance group), while 734 (92.5\%) were tolerant (tolerance group) (Fig. 1). The adverse effects observed in the intolerance group are summarized in Supplementary Table 1; diarrhea was the most common (59.3\%), followed by fever (22.0\%), headache (10.1\%), and skin rash (6.7\%). In addition, 46 patients showed intolerance to 1 type of 5-ASA, while 11 showed intolerance to 2 types of 5-ASA; the remaining 2 patients were intolerant to 3 types of 5-ASA (data not shown).

Twenty-two patients (37.2\%) in the intolerance group and 
55 patients $(7.4 \%)$ in the tolerance group were hospitalized within the 12-month observation period. The admission rate

Table 1. Baseline Characteristics of Enrolled Patients

\begin{tabular}{lcc}
\hline Characteristic & $\begin{array}{c}\text { 5-ASA intolerance } \\
(\mathrm{n}=59)\end{array}$ & $\begin{array}{c}\text { 5-ASA tolerance } \\
(\mathrm{n}=734)\end{array}$ \\
\hline Age $(\mathrm{yr})$ & $39.0 \pm 13.8$ & $46.0 \pm 14.7$ \\
Sex, male/female & $31(52.5) / 28(47.5)$ & $386(52.6) / 348(47.4)$ \\
Duration of disease $(\mathrm{yr})$ & $8.2 \pm 6.2$ & $13.1 \pm 9.1$ \\
Extent of disease, E\&L/R & $49(83.0) / 10(16.9)$ & $630(85.8) / 104(14.1)$ \\
Albumin level (g/dL) & $4.00 \pm 0.62$ & $4.20 \pm 0.50$ \\
Concomitant therapy & & \\
5-ASA & $26(44.0)$ & $725(98.7)$ \\
IM & $22(37.2)$ & $206(28.0)$ \\
Calcineurin inhibitor & $6(10.1)$ & $19(2.5)$ \\
Anti-TNF- $\alpha$ inhibitors & $13(22.0)$ & $100(13.6)$ \\
Cytapheresis & $2(3.3)$ & $21(2.8)$ \\
Total colectomy & 0 & $1(0.01)$ \\
\hline
\end{tabular}

Values are presented as mean \pm SD or number (\%).

$E \& L$, extensive colitis and left-sided colitis; $R$, proctitis; 5-ASA, 5aminosalicylic acid; IM, immunomodulator.
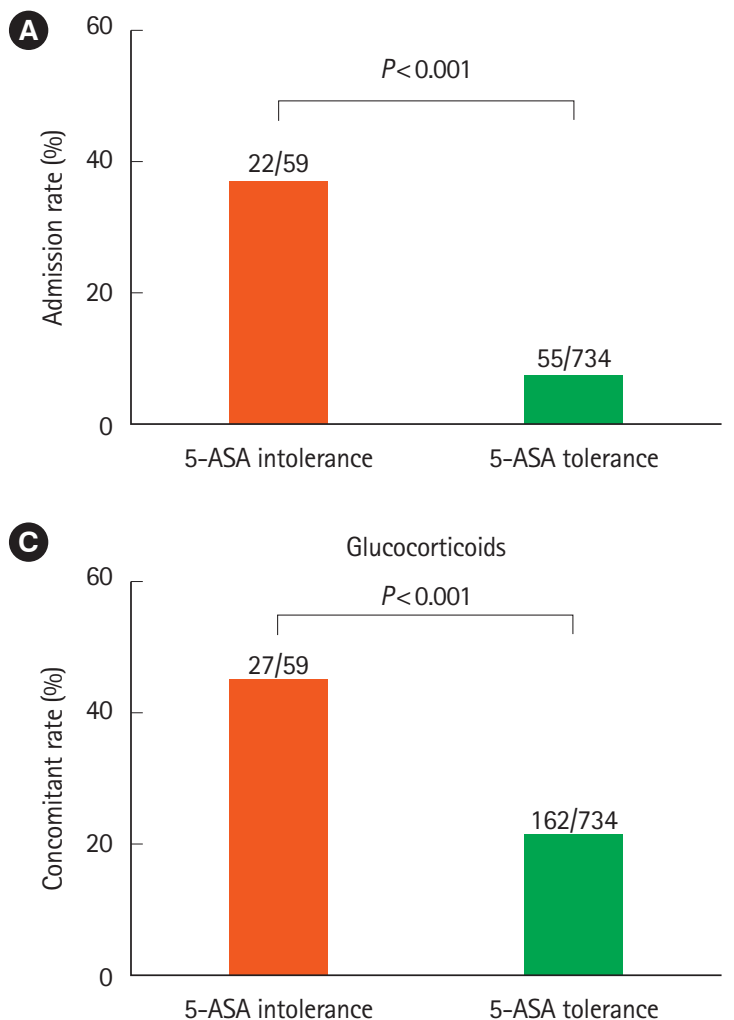

was significantly higher in the tolerance than tolerance group $(P<0.001)$ (Fig. 2A). In addition, the incidence of corticosteroid use $(P<0.001)$ and calcineurin inhibitor use $(P=0.007)$ within the observation period was significantly higher in the intolerance than tolerance group, but the incidences of IM use, anti-TNF- $\alpha$ antibody, and cytapheresis use were similar between the 2 groups (Fig. 2B-D). In the multivariate analysis, the factors significantly correlated with hospitalization were 5-ASA intolerance (OR, 7.810; 95\% CI, 3.719-16.410), extent of disease (total colitis or left-sided colitis) (OR, 10.660; 95\% CI, 1.402-81.130), and serum albumin level (OR, 0.127; 95\% CI, 0.075-0.215). Age, disease duration, and IM intolerance were not correlated with hospitalization (Table 2). These results indicate that 5-ASA intolerance significantly increased the risk of the requirement for induction therapy and hospitalization in patients with UC.

\section{5-ASA Intolerance Increases the Risk of Adverse Clinical Outcomes}

In the Keio IBD Longitudinal Cohort, 108 patients were undergoing treatment with anti-TNF- $\alpha$ biologics. The baseline
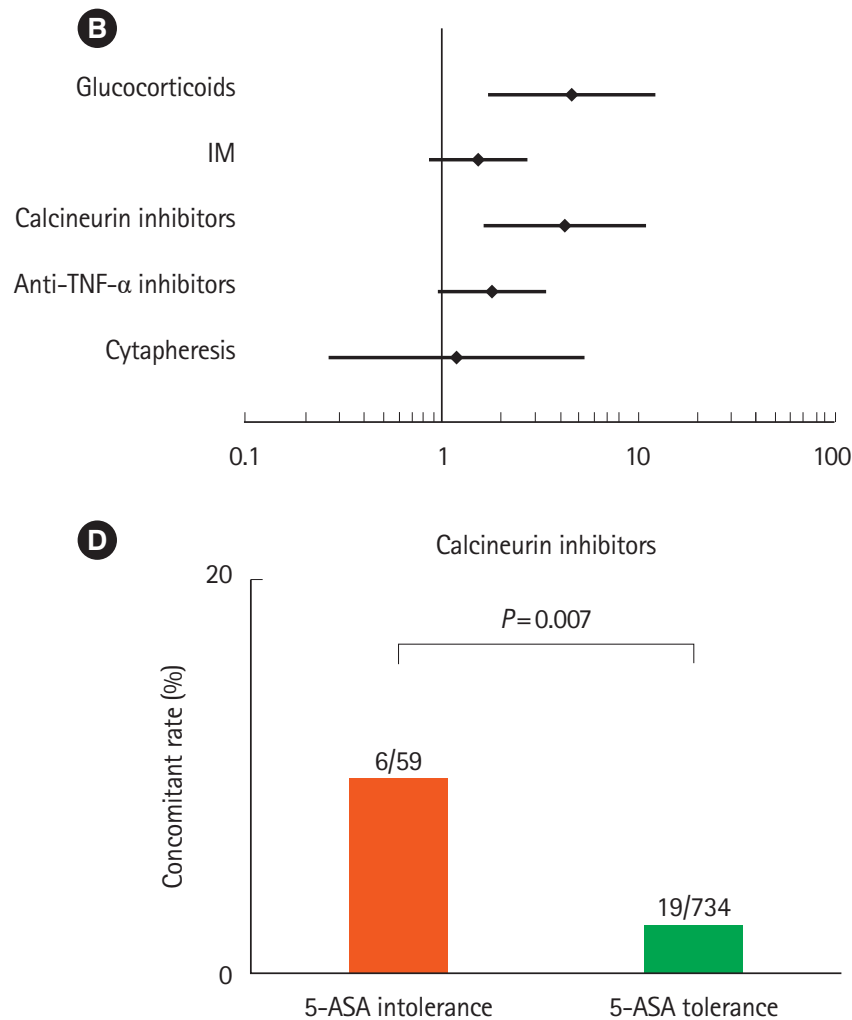

Fig. 2. Risk analysis of 5-aminosalicylic acid (5-ASA) intolerance group compared with 5-ASA tolerance group. (A) Comparison of admission rate. (B) Forest plot of univariate analysis of drug-use risk. (C) Comparison of corticosteroid use. (D) Comparison of calcineurin inhibitor use. IM, immunomodulator. 
Table 2. Risk Factors for Hospitalization in All 793 Patients

\begin{tabular}{|c|c|c|c|c|c|}
\hline \multirow{2}{*}{ Risk factor } & \multirow{2}{*}{$\begin{array}{l}\text { Admission } \\
(n=77)\end{array}$} & \multirow{2}{*}{$\begin{array}{l}\text { No admission } \\
\quad(n=716)\end{array}$} & \multirow{2}{*}{$\frac{\text { Univariate analysis }}{P \text {-value }}$} & \multicolumn{2}{|c|}{ Multivariate analysis } \\
\hline & & & & OR $(95 \% \mathrm{Cl})$ & $P$-value \\
\hline Age (yr) & $42.5 \pm 16.4$ & $45.8 \pm 14.5$ & 0.083 & - & 0.555 \\
\hline Duration of disease (yr) & $9.0 \pm 8.4$ & $13.1 \pm 9.0$ & $<0.001$ & - & 0.276 \\
\hline Extent of disease, E\&L/R & $76(98.7) / 1(1.2)$ & $603(84.2) / 113(15.7)$ & 0.001 & $10.660(1.402-81.130)$ & 0.022 \\
\hline Albumin level $(\mathrm{g} / \mathrm{dL})$ & $3.61 \pm 0.85$ & $4.28 \pm 0.41$ & $<0.001$ & $0.127(0.075-0.215)$ & $<0.001$ \\
\hline 5-ASA intolerance & $22(28.5)$ & $37(5.1)$ & $<0.001$ & $7.810(3.719-16.410)$ & $<0.001$ \\
\hline IM intolerance & $3(3.8)$ & $11(1.5)$ & 0.100 & - & 0.952 \\
\hline
\end{tabular}

Values are presented as mean \pm SD or number (\%).

E\&L, extensive colitis and left-sided colitis; R, proctitis; Alb, albumin; 5-ASA, 5-aminosalicylic acid; IM, immunomodulator.

A

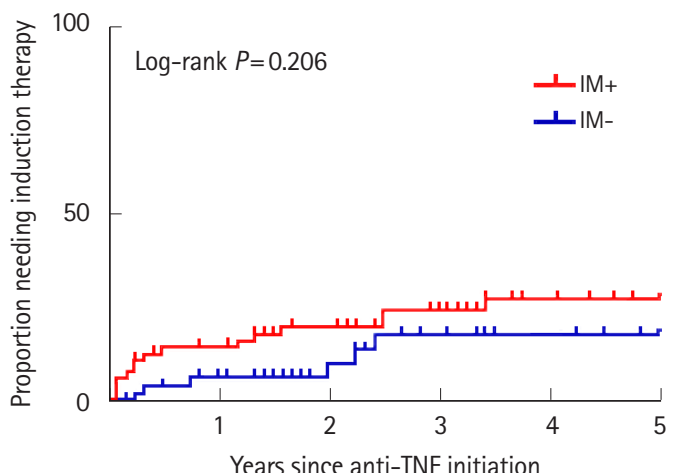

No. at risk

With IM

Without IM

$\begin{array}{llllll}62 & 50 & 39 & 30 & 19 & 14 \\ 46 & 37 & 25 & 18 & 14 & 10\end{array}$

B

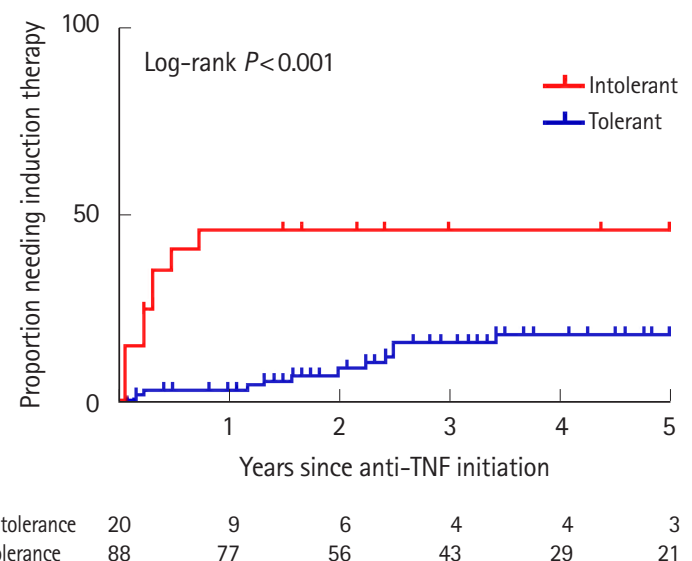

Fig. 3. Proportion of patients with UC requiring induction therapy. (A) Kaplan-Meier curve of patients with or without immunomodulator (IM) use. (B) Kaplan-Meier curve of 5-aminosalicylic acid (5-ASA)-intolerant patients and 5-ASA-tolerant patients.

patient characteristics at the start of anti-TNF- $\alpha$ therapy are shown in Supplementary Tables 2 and 3. The therapy was discontinued in 19 patients (surgical therapy, $\mathrm{n}=1$; moved to another hospital, $n=5$; adverse events, $n=5$; loss of response, $\mathrm{n}=5$; other causes, $\mathrm{n}=3$ ). The primary outcome was the need for additional induction therapy during the first 5 years after starting biologics. The study period was complete before 65 patients reached the 5-year observation point. The average observation period was 3.05 years. In the univariable analysis, concomitant IM use in patients with UC starting biologics did not decrease the risk of adverse clinical events $(P=0.206)$ (Fig. $3 \mathrm{~A})$ as shown in a previous study, ${ }^{16}$ however, our data showed that 5-ASA intolerance increased the risk of adverse clinical events $(P<0.001)$ (Fig. 3B). Notably, additional induction therapy was required within 1 year after starting biologics in many patients in the 5-ASA intolerance group. The multivariate analysis of the serum albumin level, concomitant IM use, and 5-ASA intolerance at the initiation of biologic administration was performed using binary logistic regression analysis. Interestingly, 5-ASA intolerance was identified as the only risk factor for needing induction therapy (OR, 3.284; 95\% CI, 1.02210.551) (Supplementary Tables 2 and 3).

\section{Dysbiosis Is Involved in 5-ASA Intolerance}

Next, we recruited 181 patients with UC to analyze the intestinal microbiota in feces and thus investigate the relationship between 5-ASA intolerance and the intestinal microbiota. We collected feces from 166 patients, and analyzed the feces of 124 patients in the remission phase, including 12 with 5-ASA intolerance, and 112 with 5-ASA tolerance (Fig. 4, Supplementary Table 4).

There was no significant difference in the diversity between these 2 groups (Fig. 5A and B). The taxonomic profiles showed that the abundance of the phylum Firmicutes was significantly higher in the intolerance than tolerance group $(P<0.001)$, while the abundance of the phylum Bacteroidetes was signifi- 


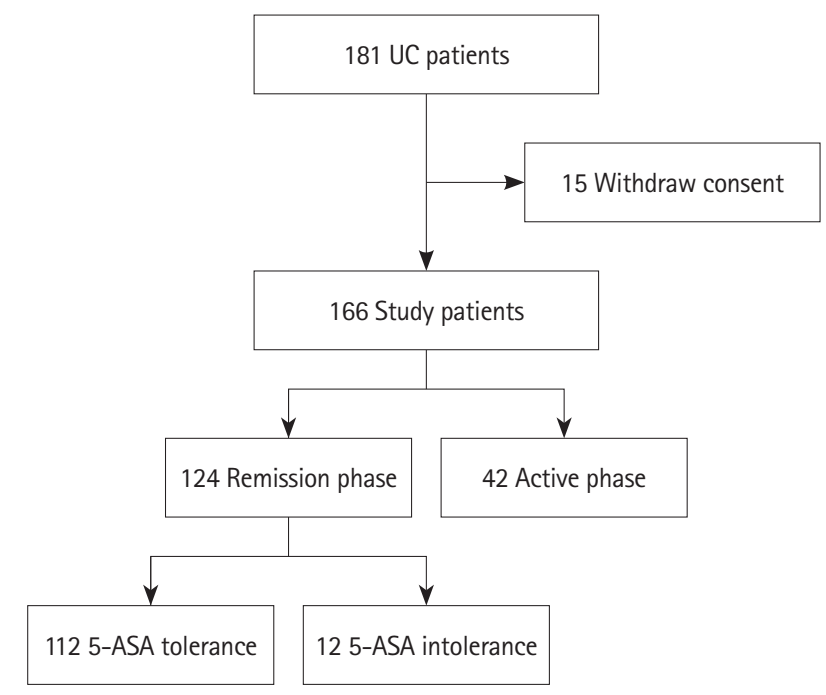

Fig. 4. Flowchart of participants in the microbial analysis. 5-ASA, 5-aminosalicylic acid.

cantly lower $(P<0.05)$ (Fig. 5C and D). The genera Faecalibacterium, Streptococcus, and Clostridium (all of which belong to the phylum Firmicutes) were significantly more abundant in the intolerance the tolerance group. Conversely, the abundance of the genus Bacteroides (which belongs to the phylum Bacteroidetes) was significantly lower in the intolerance than tolerance group $(P=0.01)$ (Fig. 5E and F). The abundance of the species Streptococcus salivarius was significantly greater in the intolerance than tolerance group $(P=0.015$; data not shown). Because of the antimicrobial activity of salazosulfapyridine (SASP), we also conducted the microbial analysis excluding patients taking SASP. The results showed almost the same trend as (Fig. 5, Supplementary Fig. 1).

Next, we observed distinct patterns at the species level between the tolerance and intolerance groups. There were no significant correlations between bacterial species in the tolerance group, while the 5-ASA intolerance group showed positive correlations between one species of Ruminococcus and Bacteroides vulgatus $(\mathrm{R}=0.92)$, and between Bifidobacterium longum and Bifidobacterium adolescentis $(\mathrm{R}=0.68)$ (Supplementary Fig. 2).

These results suggest that dysbiosis was present in the 5-ASA intolerance group.

\section{DISCUSSION}

To the best of our knowledge, the present study is the first to analyze the effect of 5-ASA intolerance on the prognosis of UC using a large-scale analysis and intestinal microbiota analysis of patients with 5-ASA intolerance. We found that 5-ASA intolerance negatively influenced the natural history of UC, and increased the risk of hospitalization and requirements for corticosteroids and calcineurin inhibitors. Previous reports showed that maintenance of long-term remission with 5-ASA reportedly reduces the risk of colorectal cancer. ${ }^{17-20}$

There is no well-established strategy for safe 5-ASA continuation in patients intolerant to 5-ASA; however, one representative method is 5-ASA desensitization. ${ }^{21}$ Desensitization is generally performed during hospitalization by starting 5-ASA at a small dosage and gradually increasing the dose. ${ }^{22,23}$ This treatment method is begun while the patient is still in the hospital because it enables prevention of emergency hospitalization and provision of adequate care for unexpected serious adverse events; furthermore, the effectiveness of desensitization remains controversial. ${ }^{24}$ In the present study, we aimed to begin the development of alternative therapeutic strategies for 5-ASA continuation in 5-ASA-intolerant patients. The intestinal microbiota is deeply involved in the pathology of UC, and multiple studies have shown that patients with UC have distinct microbial patterns compared with healthy controls. ${ }^{25,26}$ Surprisingly, we observed that the composition of the intestinal microbiota in the 5-ASA intolerance group greatly differed from that in the 5-ASA tolerance group. This suggests that dysbiosis is involved in 5-ASA intolerance and that inducing symbiosis may resolve 5-ASA intolerance. Furthermore, 5-ASA intolerance was associated with a significant increase in Firmicutes species and a significant decrease in Bacteroides species; similar microbial shifts have been found in patients with liver cirrhosis ${ }^{27}$ and obesity. ${ }^{28,29}$ Although contradictory results have been reported regarding the Firmicutes/Bacteroidetes ratio between individuals with and without obesity, recent evidence demonstrates that the dysbiosis observed in obesity is multifactorial, with environmental contributions. A high-fat diet reportedly results in intestinal dysbiosis and subsequent dysregulated gut barrier function referred to as "leaky gut,",00-32 which is characterized by a thin mucus layer and increased intestinal permeability. Bacteria and dietary fibers reportedly contribute to the formation and degradation of the mucus layer; germ-free mice have a thinner mucus layer than normal mice, while deprivation of dietary fibers in the specific pathogen-free condition results in a reduced mucus layer accompanied by overgrowth of mucin-degrading bacteria. ${ }^{33}$ Therefore, a high-fat, low-fiber Western diet may be associated with the development of 5-ASA intolerance, and restoring the "normal" gut microbiome may correct 5-ASA intolerance. Indeed, re- 

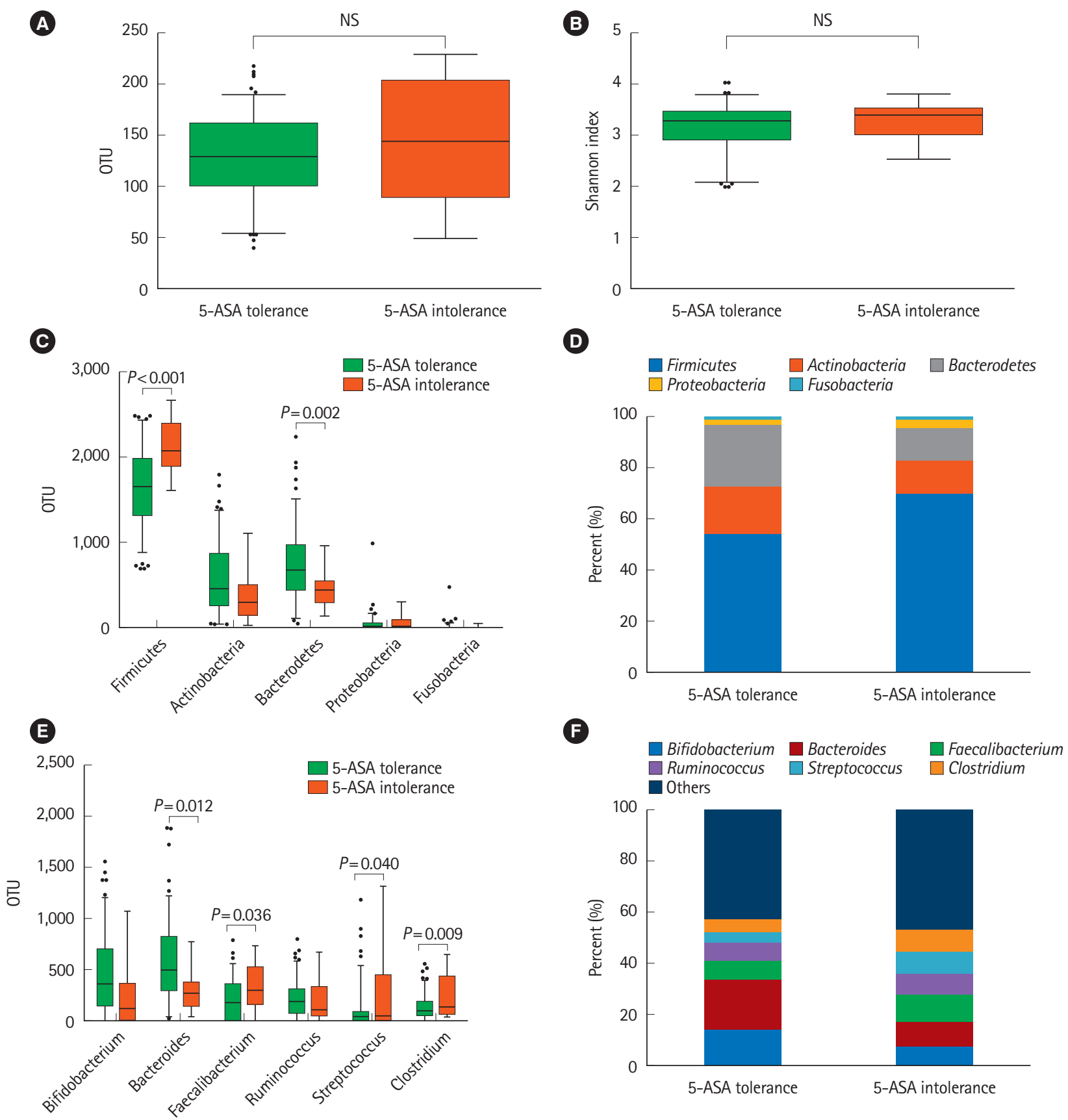

Fig. 5. Analysis of the microbiome. (A, B) Microbiota diversity (OTU number) of 5-ASA-tolerant patients $(n=112)$ and 5-ASA-intolerant patients $(n=12)$. (C, D) Comparison of 5 fecal bacteria at the phylum level. $(E, F)$ Comparison of the top 6 fecal bacteria at the genus level. 5-ASA, 5-aminosalicylic acid; OTU, operational taxonomic unit.

cent studies have shown that inducing symbiosis improves the clinical course of not only $\mathrm{UC}^{34-37}$ but also IBS, ${ }^{38-41}$ checkpoint-inhibitor-induced colitis recognized as immune-related adverse events in patients with cancer, ${ }^{42}$ hepatic encephalopathy, ${ }^{43}$ Parkinson's disease, ${ }^{44}$ and type 2 diabetes. ${ }^{45-48}$ Conventional methods used to induce symbiosis are administration of probiotics and dietary modification including intake of abun- dant dietary fiber; however, fecal microbiota transplantation (FMT) has attracted attention as a method for dramatically altering the intestinal microbiota. FMT reportedly has the potential to induce remission in some patients with $\mathrm{UC}^{49-52}$ and may be an effective therapeutic strategy for 5-ASA intolerance. Further clinical and basic studies are needed to examine the efficacy of FMT and determine role of dysbiosis in the devel- 
opment of 5-ASA intolerance.

A limitation of the present study is that there was no unified definition of 5-ASA intolerance, because this was a retrospective observational study. In addition, 5-ASA intolerance group may include the patients who have abdominal symptoms due to worsening of UC disease activity. Although the number of patients in whom the intestinal microbiota was analyzed was limited, we identified the distinct intestinal microbiome patterns associated with 5-ASA intolerance. Furthermore, since 5-ASA itself may affect the intestinal microbiota, further study is needed to show the changes in intestinal microbiota before and after 5-ASA administration and eliminate the antibacterial properties of 5-ASA.

The present study revealed that patients intolerant to 5-ASA were more likely to require hospitalization and induction therapy. Hence, it may be important to conduct therapeutic interventions earlier in patients with 5-ASA intolerance, and thus lead them into deep remission. In addition, these patients with UC with 5-ASA intolerance had a distinct fecal microbiota pattern. Despite an increasing use of biologics and small molecule inhibitors, 5-ASA remains a mainstay of maintenance therapy for patients with UC. It is expected that it will be difficult to maintain intestinal homeostasis in 5-ASA intolerance group, and there are cases of loss of response to biologics. Therefore, it is speculated that there are many cases that require induction therapy in patients with 5-ASA intolerance. Our data suggest that dysbiosis may influence the development of 5-ASA intolerance. Future studies will identify which species or strains of the microbiota cause 5-ASA intolerance in more detail and clarify whether the microbiota is a biomarker of 5-ASA intolerance. These future studies may prove whether correcting the microbiota prevents or reverses 5-ASA intolerance.

\section{FINANCIAL SUPPORT}

This work was supported by the Japan Agency for Medical Research and Development (16gm1010003h0001) (Kanai T), JSPS KAKENHI (grant No. 17K15966) (Mizuno S), and Takeda Science Foundation (Mizuno S).

\section{CONFLICT OF INTEREST}

No potential conflict of interest relevant to this article was reported.

\section{AUTHOR CONTRIBUTION}

Study concept and design: Mizuno S, Mikami Y, Naganuma M. Statistical analysis: Mizuno S, Ono K, Mikami Y, Fukuda T. Recruitment of participants and acquisition of data: Mizuno $\mathrm{S}$, Naganuma M, Terada S, Yoshida T, Saigusa K, Kanai T. Drafting of the manuscript: Mizuno S, Mikami Y, Naganuma M, Kanai T. Database construction and management: Mizuno S, Minami K, Masaoka T, Hirahara N, Miyata H. Microbial analysis: Mizuno S, Ono K, Mikami Y, Suda W, Hattori M. All authors contributed to critical review and approved the final draft.

\section{ORCID}

$\begin{array}{ll}\text { Mizuno S } & \text { https://orcid.org/0000-0002-5472-2282 } \\ \text { Mikami Y } & \text { https://orcid.org/0000-0001-5104-4415 } \\ \text { Naganuma M } & \text { https://orcid.org/0000-0002-4244-3243 } \\ \text { Fukuda T } & \text { https://orcid.org/0000-0002-6539-5002 } \\ \text { Masaoka T } & \text { https://orcid.org/0000-0002-8598-1009 } \\ \text { Hirahara N } & \text { https://orcid.org/0000-0002-8931-5927 } \\ \text { Miyata H } & \text { https://orcid.org/0000-0001-8800-6201 } \\ \text { Suda W } & \text { https://orcid.org/0000-0002-2861-9724 } \\ \text { Hattori M } & \text { https://orcid.org/0000-0001-9467-0344 } \\ \text { Kanai T } & \text { https://orcid.org/0000-0002-1466-4532 }\end{array}$

\section{SUPPLEMENTARY MATERIAL}

Supplementary materials are available at the Intestinal Research website (https://www.irjournal.org).

\section{REFERENCES}

1. Danese S, Fiocchi C. Ulcerative colitis. N Engl J Med 2011;365: 1713-1725.

2. Almario CV, Keller MS, Chen M, et al. Optimizing selection of biologics in inflammatory bowel disease: development of an online patient decision aid using conjoint analysis. Am J Gastroenterol 2018;113:58-71.

3. White JR, Phillips F, Monaghan T, et al. Review article: novel oral-targeted therapies in inflammatory bowel disease. Aliment Pharmacol Ther 2018;47:1610-1622.

4. Kaplan GG, Seow CH, Ghosh S, et al. Decreasing colectomy rates for ulcerative colitis: a population-based time trend study. Am J Gastroenterol 2012;107:1879-1887.

5. Vester-Andersen MK, Prosberg MV, Jess T, et al. Disease course and surgery rates in inflammatory bowel disease: a 
population-based, 7-year follow-up study in the era of immunomodulating therapy. Am J Gastroenterol 2014;109:705-714.

6. Pillai N, Dusheiko M, Burnand B, Pittet V. A systematic review of cost-effectiveness studies comparing conventional, biological and surgical interventions for inflammatory bowel disease. PLoS One 2017;12:e185500.

7. Ford AC, Achkar JP, Khan KJ, et al. Efficacy of 5-aminosalicylates in ulcerative colitis: systematic review and meta-analysis. Am J Gastroenterol 2011;106:601-616.

8. Di Paolo MC, Paoluzi OA, Pica R, et al. Sulphasalazine and 5-aminosalicylic acid in long-term treatment of ulcerative colitis: report on tolerance and side-effects. Dig Liver Dis 2001;33:563-569.

9. Ransford RA, Langman MJ. Sulphasalazine and mesalazine: serious adverse reactions re-evaluated on the basis of suspected adverse reaction reports to the Committee on Safety of Medicines. Gut 2002;51:536-539.

10. Dignass A, Van Assche G, Lindsay JO, et al. The second European evidence-based consensus on the diagnosis and management of Crohn's disease: current management. J Crohns Colitis 2010;4:28-62.

11. Wang Y, Parker CE, Feagan BG, MacDonald JK. Oral 5-aminosalicylic acid for maintenance of remission in ulcerative colitis. Cochrane Database Syst Rev 2016;(5):CD000544.

12. Bernstein CN, Forbes JD. Gut microbiome in inflammatory bowel disease and other chronic immune-mediated inflammatory diseases. Inflamm Intest Dis 2017;2:116-123.

13. Zhang M, Sun K, Wu Y, Yang Y, Tso P, Wu Z. Interactions between intestinal microbiota and host immune response in inflammatory bowel disease. Front Immunol 2017;8:942.

14. Shimizu H, Arai K, Tang J, Hosoi K, Funayama R. 5-Aminosalicylate intolerance causing exacerbation in pediatric ulcerative colitis. Pediatr Int 2017;59:583-587.

15. Nishijima S, Suda W, Oshima K, et al. The gut microbiome of healthy Japanese and its microbial and functional uniqueness. DNA Res 2016;23:125-133.

16. Ungaro RC, Limketkai BN, Jensen CB, et al. Stopping 5-aminosalicylates in patients with ulcerative colitis starting biologic therapy does not increase the risk of adverse clinical outcomes: analysis of two nationwide population-based cohorts. Gut 2019;68:977-984.

17. Bonovas S, Fiorino G, Lytras T, Nikolopoulos G, Peyrin-Biroulet L, Danese S. Systematic review with meta-analysis: use of 5-aminosalicylates and risk of colorectal neoplasia in patients with inflammatory bowel disease. Aliment Pharmacol Ther 2017;45:1179-1192.
18. Harbord M, Eliakim R, Bettenworth D, et al. Third European evidence-based consensus on diagnosis and management of ulcerative colitis. part 2: current management. J Crohns Colitis 2017;11:769-784.

19. Velayos FS, Terdiman JP, Walsh JM. Effect of 5-aminosalicylate use on colorectal cancer and dysplasia risk: a systematic review and metaanalysis of observational studies. Am J Gastroenterol 2005;100:1345-1353.

20. Zhao LN, Li JY, Yu T, Chen GC, Yuan YH, Chen QK. 5-Aminosalicylates reduce the risk of colorectal neoplasia in patients with ulcerative colitis: an updated meta-analysis. PLoS One 2014;9:e94208.

21. Tolia V. Sulfasalazine desensitization in children and adolescents with chronic inflammatory bowel disease. Am J Gastroenterol 1992;87:1029-1032.

22. Heath JL, Heath RD, Tamboli C, et al. Mesalamine desensitization in a patient with treatment refractory ulcerative colitis and aspirin and nonsteroidal anti-inflammatory drug hypersensitivity. Ann Allergy Asthma Immunol 2017;118:518-520.

23. Panganiban CM, Pourang D, Samant SA. A novel 2-day desensitization protocol to oral mesalamine. J Allergy Clin Immunol Pract 2018;6:695-696.

24. Buurman DJ, De Monchy JG, Schellekens RC, van der Waaij LA, Kleibeuker JH, Dijkstra G. Ulcerative colitis patients with an inflammatory response upon mesalazine cannot be desensitized: a randomized study. Scand J Gastroenterol 2015; 50:399-405.

25. Machiels K, Joossens M, Sabino J, et al. A decrease of the butyrate-producing species Roseburia hominis and Faecalibacterium prausnitzii defines dysbiosis in patients with ulcerative colitis. Gut 2014;63:1275-1283.

26. Noor SO, Ridgway K, Scovell L, et al. Ulcerative colitis and irritable bowel patients exhibit distinct abnormalities of the gut microbiota. BMC Gastroenterol 2010;10:134.

27. Qin N, Yang F, Li A, et al. Alterations of the human gut microbiome in liver cirrhosis. Nature 2014;513:59-64.

28. Ley RE, Turnbaugh PJ, Klein S, Gordon JI. Microbial ecology: human gut microbes associated with obesity. Nature 2006; 444:1022-1023.

29. Koliada A, Syzenko G, Moseiko V, et al. Association between body mass index and Firmicutes/Bacteroidetes ratio in an adult Ukrainian population. BMC Microbiol 2017;17:120.

30. Claesson MJ, Jeffery IB, Conde S, et al. Gut microbiota composition correlates with diet and health in the elderly. Nature 2012;488:178-184

31. Hermiston ML, Gordon JI. Inflammatory bowel disease and 
adenomas in mice expressing a dominant negative N-cadherin. Science 1995;270:1203-1207.

32. Ley RE, Bäckhed F, Turnbaugh P, Lozupone CA, Knight RD, Gordon JI. Obesity alters gut microbial ecology. Proc Natl Acad Sci U S A 2005;102:11070-11075.

33. Desai MS, Seekatz AM, Koropatkin NM, et al. A dietary fiberdeprived gut microbiota degrades the colonic mucus barrier and enhances pathogen susceptibility. Cell 2016;167:13391353.

34. Damman CJ, Miller SI, Surawicz CM, Zisman TL. The microbiome and inflammatory bowel disease: is there a therapeutic role for fecal microbiota transplantation? Am J Gastroenterol 2012;107:1452-1459.

35. Derwa Y, Gracie DJ, Hamlin PJ, Ford AC. Systematic review with meta-analysis: the efficacy of probiotics in inflammatory bowel disease. Aliment Pharmacol Ther 2017;46:389-400.

36. Koretz RL. Probiotics in gastroenterology: how pro is the evidence in adults? Am J Gastroenterol 2018;113:1125-1136.

37. McIlroy J, Ianiro G, Mukhopadhya I, Hansen R, Hold GL. Review article: the gut microbiome in inflammatory bowel disease-avenues for microbial management. Aliment Pharmacol Ther 2018;47:26-42

38. Halkjær SI, Christensen AH, Lo BZS, Browne PD, Günther S, Hansen LH, et al. Faecal microbiota transplantation alters gut microbiota in patients with irritable bowel syndrome: results from a randomised, double-blind placebo-controlled study. Gut 2018;67:2107-2115.

39. Johnsen PH, Hilpüsch F, Cavanagh JP, et al. Faecal microbiota transplantation versus placebo for moderate-to-severe irritable bowel syndrome: a double-blind, randomised, placebocontrolled, parallel-group, single-centre trial. Lancet Gastroenterol Hepatol 2018;3:17-24.

40. Kurokawa S, Kishimoto T, Mizuno S, et al. The effect of fecal microbiota transplantation on psychiatric symptoms among patients with irritable bowel syndrome, functional diarrhea and functional constipation: an open-label observational study. J Affect Disord 2018;235:506-512.

41. Mizuno S, Masaoka T, Naganuma M, et al. Bifidobacteriumrich fecal donor may be a positive predictor for successful fecal microbiota transplantation in patients with irritable bowel syndrome. Digestion 2017;96:29-38.
42. Dubin K, Callahan MK, Ren B, et al. Intestinal microbiome analyses identify melanoma patients at risk for checkpointblockade-induced colitis. Nat Commun 2016;7:10391.

43. Bajaj JS, Kassam Z, Fagan A, et al. Fecal microbiota transplant from a rational stool donor improves hepatic encephalopathy: a randomized clinical trial. Hepatology 2017;66:17271738.

44. Evrensel A, Ceylan ME. Fecal microbiota transplantation and its usage in neuropsychiatric disorders. Clin Psychopharmacol Neurosci 2016;14:231-237.

45. Bouter KE, van Raalte DH, Groen AK, Nieuwdorp M. Role of the gut microbiome in the pathogenesis of obesity and obesity-related metabolic dysfunction. Gastroenterology 2017;152: 1671-1678.

46. Hampe CS, Roth CL. Probiotic strains and mechanistic insights for the treatment of type 2 diabetes. Endocrine 2017;58: 207-227.

47. Wu H, Esteve E, Tremaroli V, et al. Metformin alters the gut microbiome of individuals with treatment-naive type 2 diabetes, contributing to the therapeutic effects of the drug. Nat Med 2017;23:850-858.

48. Zhu A, Chen J, Wu P, et al. Cationic polystyrene resolves nonalcoholic steatohepatitis, obesity, and metabolic disorders by promoting eubiosis of gut microbiota and decreasing endotoxemia. Diabetes 2017;66:2137-2143.

49. Costello SP, Soo W, Bryant RV, Jairath V, Hart AL, Andrews JM. Systematic review with meta-analysis: faecal microbiota transplantation for the induction of remission for active ulcerative colitis. Aliment Pharmacol Ther 2017;46:213-224.

50. Moayyedi P, Surette MG, Kim PT, et al. Fecal microbiota transplantation induces remission in patients with active ulcerative colitis in a randomized controlled trial. Gastroenterology 2015;149:102-109.

51. Narula N, Kassam Z, Yuan Y, et al. Systematic review and meta-analysis: fecal microbiota transplantation for treatment of active ulcerative colitis. Inflamm Bowel Dis 2017;23:17021709.

52. Paramsothy S, Kamm MA, Kaakoush NO, et al. Multidonor intensive faecal microbiota transplantation for active ulcerative colitis: a randomised placebo-controlled trial. Lancet 2017;389: 1218-1228. 


\section{See "5-Aminosalicylic acid intolerance is associated with a risk of adverse clinical outcomes and dysbiosis in patients with ulcerative colitis" on page 69-78.}

Supplementary Table 1. Adverse Effects Experienced by Patients Intolerant to 5-ASA

\begin{tabular}{lc}
\hline Side effects to 5-ASA & No. $(\%)(n=59)$ \\
\hline Diarrhea & $35(59.3)$ \\
Fever & $13(22.0)$ \\
Headache & $6(10.1)$ \\
Rash & $4(6.7)$ \\
Abdominal pain & $2(3.3)$ \\
Others & $7(11.8)$ \\
\hline
\end{tabular}

ASA, aminosalicylic acid.

Supplementary Table 3. Risk Factors for Additional Treatment

\begin{tabular}{|c|c|c|c|c|c|}
\hline \multirow{2}{*}{ Risk factor } & \multirow{2}{*}{$\begin{array}{l}\text { Additional treatment } \\
\qquad(n=23)\end{array}$} & \multirow{2}{*}{$\begin{array}{l}\text { No additional treatment } \\
\qquad(n=85)\end{array}$} & \multirow{2}{*}{$\frac{\text { Univariate analysis }}{P \text {-value }}$} & \multicolumn{2}{|c|}{ Multivariate analysis } \\
\hline & & & & OR $(95 \% \mathrm{Cl})$ & $P$-value \\
\hline Albumin level (g/dL) & $3.68 \pm 0.64$ & $3.56 \pm 0.72$ & 0.517 & - & 0.767 \\
\hline 5-ASA intolerance & $9(39.1)$ & $11(12.9)$ & 0.025 & $3.284(1.022-10.551)$ & 0.046 \\
\hline Concomitant IM & 15 (65.2) & 47 (55.2) & 0.469 & - & 0.763 \\
\hline
\end{tabular}

Values are presented as mean \pm SD or number (\%).

ASA, aminosalicylic acid; IM, immunomodulator. 
Supplementary Table 4. Baseline Characteristics of Patients Included in the Intestinal Microbiota Analysis

\begin{tabular}{|c|c|c|c|}
\hline Characteristic & $\begin{array}{l}\text { 5-ASA tolerance } \\
(n=112)\end{array}$ & $\begin{array}{l}\text { 5-ASA intolerance } \\
\quad(n=12)\end{array}$ & $P$-value \\
\hline Age (yr) & $46.4 \pm 13.2$ & $50.3 \pm 14.9$ & 0.35 \\
\hline Sex, male/female & $64(57.1) / 48$ (42.9) & $6(50.0) / 6(50.0)$ & 0.76 \\
\hline Duration of disease (yr) & $14.1 \pm 9.3$ & $9.2 \pm 6.7$ & 0.09 \\
\hline Extent of disease, E\&L/R & $90(80.4) / 22(19.6)$ & $8(66.7) / 4(33.3)$ & 0.27 \\
\hline Albumin level (g/dL) & $4.5 \pm 0.4$ & $4.4 \pm 0.3$ & \\
\hline \multicolumn{4}{|l|}{ Concomitant therapy } \\
\hline 5-ASA & $107(95.5)$ & $6(50.0)$ & 0.01 \\
\hline $\mathrm{IM}$ & 47 (42.0) & $5(41.7)$ & 0.99 \\
\hline Calcineurin inhibitors & 0 & 0 & ND \\
\hline Anti-TNF- $\alpha$ inhibitors & $10(8.9)$ & $3(25.0)$ & 0.11 \\
\hline Cytapheresis & 0 & 0 & ND \\
\hline Total colectomy & 0 & 0 & ND \\
\hline
\end{tabular}

Values are presented as mean \pm SD or number (\%).

ASA, aminosalicylic acid; E\&L, extensive colitis and left-sided colitis; R, proctitis; IM, immunomodulator; ND, not determined.

A

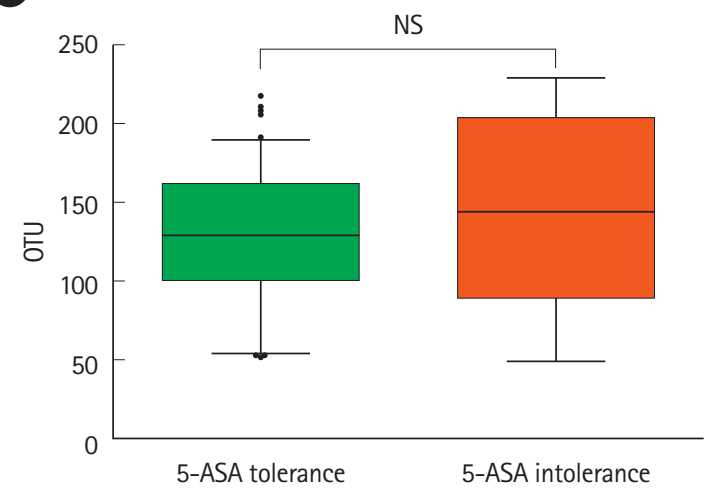

C

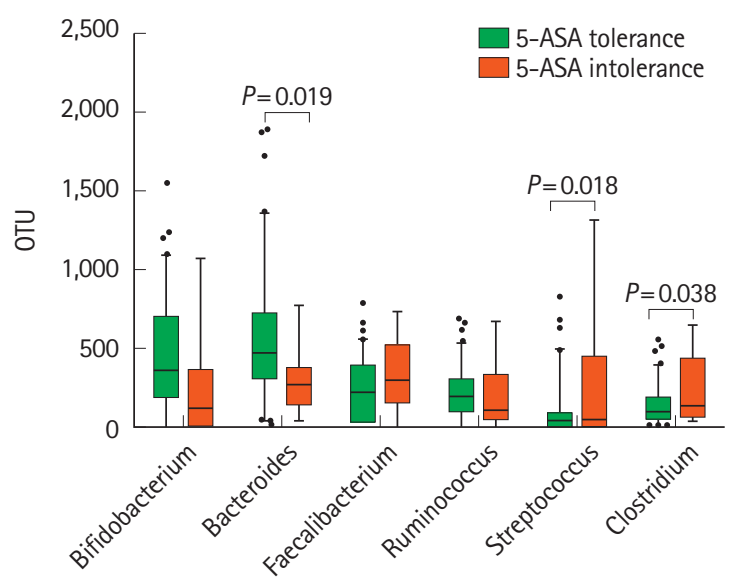

B

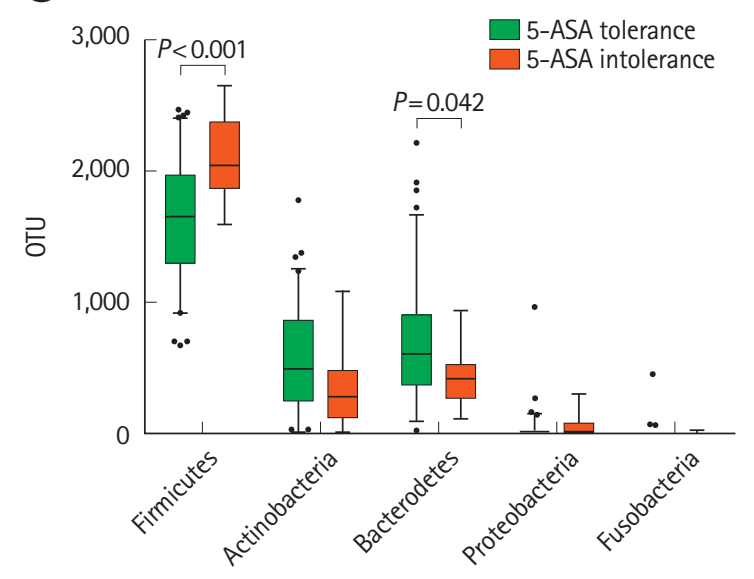

Supplementary Fig. 1. Analysis of the microbiome excluding patients taking salazosulfapyridine. (A) Microbiota diversity (OTU number) of 5-ASA-tolerant patients $(n=81)$ and 5-ASA-intolerant patients $(n=12)$. (B) Comparison of 5 fecal bacteria at the phylum level. (C) Comparison of the top 6 fecal bacteria at the genus level. 5-ASA, 5-aminosalicylic acid; OTU, operational taxonomic unit. 
(A)

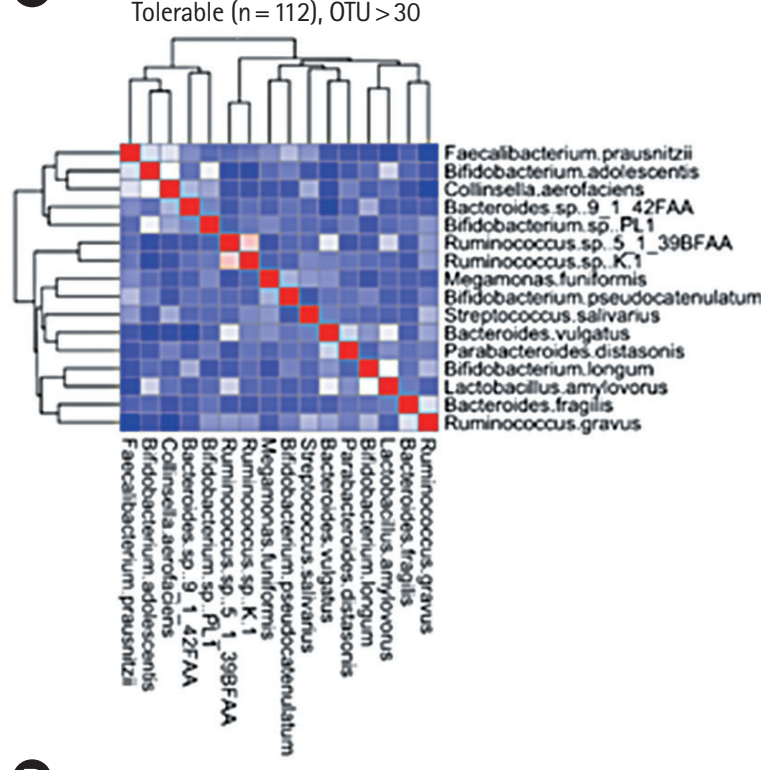

B

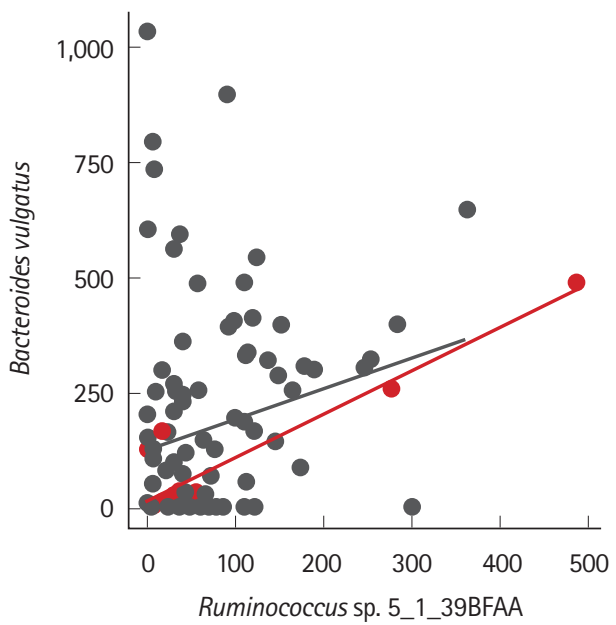

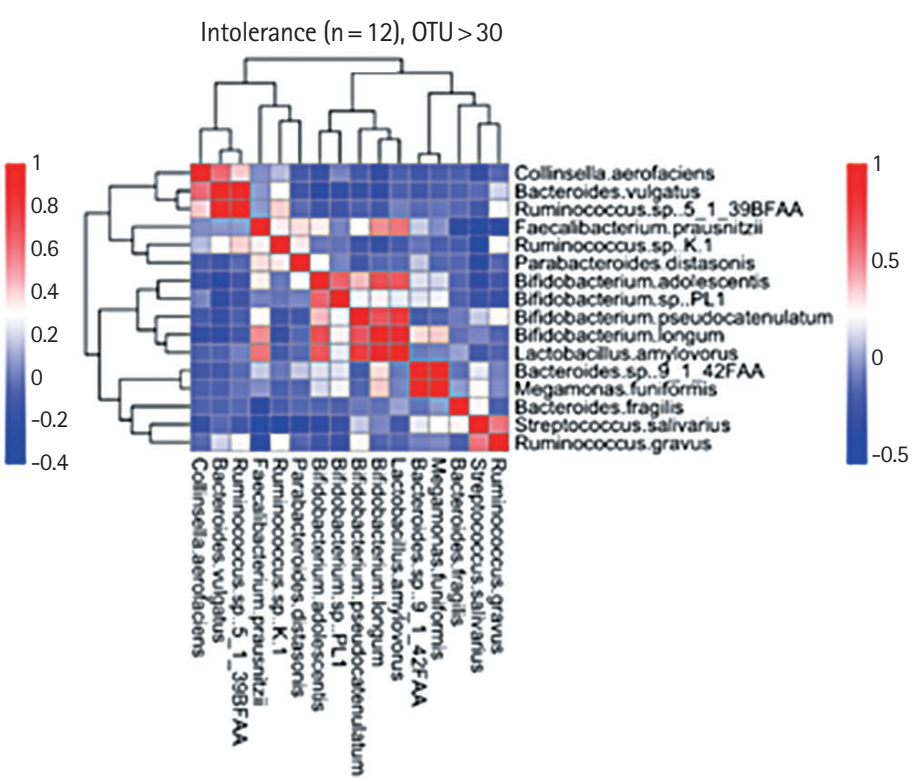

C

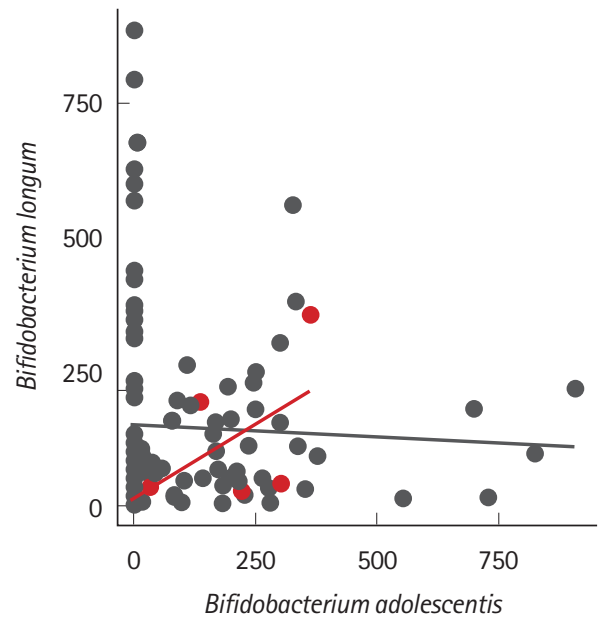

Supplementary Fig. 2. (A) Heat map analysis showing the Pearson correlation coefficients for the comparisons between each species (left: tolerance group and right: intolerance group). (B, C) Scatter plot for correlation analysis. The red line and dots represent the 5-aminosalicylic acid (5-ASA) intolerance group. The gray line and dots represent the 5-ASA tolerance group. OTU, operational taxonomic unit. 